\title{
Investigation and Application Progress of Vero Cell Serum-free Culture
}

\author{
Tian Chen \& Keping Chen \\ School of life science, Jiangsu University, Jiangsu 212003, China \\ E-mail: chenlpwx@163.com
}

\begin{abstract}
Serum-free culture is now becoming the general trend of biological production. Its application in Vero cell culture is significant. In the present paper, we reviewed factors affecting Vero cell serum-free culture and several culture techniques. In light of these reviews, we outlined its extensive application prospect.
\end{abstract}

Keywords: Vero cell, Vaccine, Serum-free, Microcarrier

\section{Background of Vero cell serum-free culture}

Vero cells are considered as a substrate for the production of viral vaccines. Vero cells were first separated from a normal adult African green monkey kidney cell line by Yasumura of Chiba University, Japan in 1962. This cell line presents several advantages over primary and diploid cell substrates. (1) Vero cells are easily available, grow fast and require no rigor culture conditions which can support various virus proliferations; (2)Vero cells have been extensively characterized to evaluate their oncogenic potential. Several studies have shown that this cell line is free from oncogenic property and is not presenting any threat to human health when used as a substrate for biological production authorized by the World Health Organization (WHO); (3)Vero cells can be used in microcarrier and suspension cultures for large scale production in bioreactors. Moreover, virus titer achieved is higher than that reached using other types of cell substrates. These properties greatly facilitate the transfer of vaccine-producing capability to developing countries, which is an important goal of WHO.

Cell cultures have been used extensively in the manufacture of biologicals for diagnostics, therapeutics and vaccines. The production of vaccines by animal cell culture was one of the earliest commercial applications of in vitro animal cell technology, and despite the recent spotlight accorded recombinant protein products, vaccines remain the most significant application of cell culture in terms of product numbers and social impact. Product safety is of paramount concern since the majority of viral vaccines are for use in healthy paediatrics. Cell substrate, production cultures, culture conditions, production media, detachment agents, etc. are major issues of safety considerations (Rourou, 2007). One of the important points of safety concerns relates to the composition of culture medium. All classical vaccine production processes make use of animal derived substances: serum, trypsin, lactalbumin, etc. Animal sera are required for vigorous growth of animal cells used as substrate for virus propagation. Serum was shown to have several essential functions in culture. It is a source of nutrients, hormones, growth factors and protease inhibitors. Furthermore, serum facilitates the attachment and spreading of cells, and provides nonspecific protection against mechanical damage and shear forces. In addition, it is able to bind to toxic compounds. However, besides these growth-promoting properties, serum has some major disadvantages. It is undefined with respect to its chemical composition. It can be a source of adventitious agents and their by-products. Serum also presents a variable performance of cell growth and has a substantial cost. Therefore, the benefits of in vitro culture of animal cell lines in the absence of serum are widely acknowledged. The removal of serum components and the elimination of other animal-derived raw materials are proving more challenging. Processes for serum free culture of several cell types expressing r-DNA derived proteins and monoclonal antibodies (MAbs) have been developed and established for large scale production since the beginning of the 1990s. However, the picture is different for existing conventional biotechnology products such as viral vaccines, the scientific challenge is probably greater because diploid cell lines or even continuous cell lines (CCLs) such as Vero cells may require additional growth and attachment factors that CCLs growing in suspension culture do not need. Serum free culture applied in production area would give greater application potential and broad market prospect Vero cells. Serum free culture of Vero cells has been an important focus on produce and research currently or for the future. 


\section{Studies on serum free culture of Vero cells}

\subsection{Serum free medium}

Medium is the most direct and paramount environmental factor affecting growth metabolism, proliferation and survival of cells in vitro. Hence, the key point of serum free culture is the optimization and development of serum free medium. For many years, whether small scale laboratory or large scale medium producer, whether for scientific research or mass production, more and more attention have been paid to the studies and researches on the serum free medium of Vero cells and thus corresponding improvements have been continuously made.

\subsubsection{Independent development}

There are two ways to develop serum free medium: one is to design and assay bran-new medium suitable for cell proliferation. In the method, components were gradually changed one by one according to the known formula of certain cell lines until attaining optimized in line with particular requirement. A detailed growth curve and clonal growth should be undertaken at the expense of time and labor; The other is to add several possible conducive components to assure the normal growth with reference to available formula and gained experience, and on this basis, eliminate toxic or void components and optimize several components to advisable concentration. Numerous statistic methods are applied in the method due to the complicated constitute of medium and many affecting factors. Mass production mainly relied on the serum free medium formula developed in the combination of certain cell lines and produce technology by research department of biological pharmacy enterprises, and contract production of medium manufacturer; Small scale production relied on medium formula confirmed by research institutions and produce enterprises and adding several serum substitute to the commercial synthetic medium, which was an economic way at low cost. Currently, based on commercial medium such as DMEM, F-12, M199, RPMI 1640, etc., serum free medium of Vero cells used either or in the combination of them, added some trace elements, growth factor, hormone, transport protein and adhesion factor, increased or decreased amino acid and the concentration of lipids and its precursor. Statistic method and software were applied to optimize the ration of each component and make the good use of them.

Gao and Wei (Hong-liang and Wei, 2001) have found that amino acids are very important nutrients in mammalian cell cultivation, 12 amino acids (Gln, Arg, His, Leu, Thr, Ile, Lys, Ser, Tyr, Phe, Val and Met) were consumed, and 4 amino acids (Glu, Asp, Ala and Pro) were produced during the growth of Vero cells. Amino acid Gln and Ser should be first added into the serum free medium because Sebastien (Sebastien Quesney, 2001)have pointed out that they are the first deficient components in the medium and possibly main factors inducing cell apoptosis. Adhesion factor added are mainly components of intercellular substance and serum, such as fibronectin, collagen, and laminin. When studies of cell biology came into molecular domain, it was noted that sequence Arg-Gly-Asp (RGD) in previous extracellular matrix proteins mediated cell attachment by interacting with proteins of the integrin family of cell surface receptors. RGD promoted the attachment of Vero cells to polystyrene and cellulose acetate (Andrew Wierzba, 1995). Vero cells in the prepared serum- and protein- free medium by adding yeast extract and soy hydrolysate into basic medium grew well(Zhenglun and Xianping, 2007), for these hydrolysates contained low relative molecular mass peptides, which could afford physical protection and promote cell attachment. However, recent research has noted that great discrepancy existed in batches of these hydrolysates, which could be the new uncertain components of serum free medium (Genzel, 2006). Consequently, seeking for new, more efficient and definite additive is urgent for preparing serum free medium at present or in the future. In order to cut down cost and mass production, cheaper additive or nutrient substitutes are considered, such as chelating agents or citrates to simulate the function of transport protein, surfactants to protect fluid mapper shear for protein.

\subsubsection{Commercial product}

Due to high specificity of serum free medium, commercial serum free medium suitable for other cell lines is not desirable for Vero cells, which has been confirmed by experiments. Currently, routine Vero cell commercial serum free medium are products of high quality, specially designed for Vero cells. These products get improvements continuously in quality and quantity. (1) The most typical medium among numerous products is VP-SFM(virus production-serum free media), produced by the largest cell culture product manufacturer(GIBCO/Invitrogen). It is a serum free medium with ultra-low protein. Microprotein in VP-SFM derived from recombinant protein. Transferrin was already replaced by a kind of ion chelate, and albumin was replaced by dipeptide or tripeptide from plants. The only necessary addition is L-glutamine, with a concentration of $2 \sim 6 \mathrm{mM}$ according to the relative cell lines. Vevo cells are not necessary to adapt to VP-SFM. Cell density could attain $1.8 \times 10^{5}$ cells $/ \mathrm{cm}^{2}$ when static incubation in $75 \mathrm{~cm}^{2}$ spinner flasks. Just as its name implies, VP-SFM is kind of medium particular for the application of virus propagation. Many reports of VP-SFM used for producing virus are available(Frazzati-Gallina, 2001; Toriniwa, 2007). (2)OptiPro SFM is another serum free medium of GIBCO/Invitrogen for Vero cells, with uncertain chemical components and no peptides from plants, animals or synthetical protein, excluding Vero cells, and is also suitable for kidney derived cells such as MDBK, BHK-21, PK-15 and so on with respect to virus producing. For attachment dependent cells, it is unnecessary to add some attachment protein or pretreatment with culture surface. (3)EX-CELL ${ }^{\mathrm{TM}}$ VERO produced by JRH, is a kind of serum 
free medium specially designed for the high density growth of Vero cells, supporting attachment and suspension culture. It contains no animal components and hydrolysates from plants, with low level of recombinant protein and no phenol red or PluronicF68. When EX-CELL ${ }^{\mathrm{TM}}$ VERO transferred from serum containing medium, cells need a short period to accommodate, compared to other similar products, the particular dominance is the high density growth supporting spinner flask static culture and microcarrier culture in bioreactor. Cell density could attain $2 \times 10^{5} \mathrm{cell} / \mathrm{s} / \mathrm{cm}^{2}$ when static incubation in $75 \mathrm{~cm}^{2}$ spinner flasks and $1.9 \times 10^{6} \mathrm{cells} / \mathrm{ml}$ in bioreactor with microcarrier Cytodex-1. In serum free culture, cell density and virus titers was identical to the level in serum containing culture (Kolell, 2005).

Except the above-mentioned mediums, commercial serum free mediums for Vero cells include MD Cell DMEM (Biowest), MP-Vero ${ }^{\mathrm{TM}}$ SFM (MP), Plus VEROCesco Bioengineering Co., Hsinchu, Taiwan(CESCO), ICN-VERO(ICN), PEEK-1(Biochrom) and so on. But reports on them are less available.

Main issues existed in the commercial serum free medium are as follows: They could only be available in the form of liquid; They are expensive with unknown components due to severe commercial security; They are only suitable for laboratory or small scale investigation, and not acceptable due to its high cost in industrial mass production.

\subsection{Serum free culture}

\subsubsection{Culture mode}

\section{(1) Attachment culture}

Vero cells, as well as other many cell lines with great application value in industry (including BHK, CHO and 293 cells), are all attachment dependent. Vero cells have experienced a development process from experimental spinner bottles to small industrial scale roller bottles, however, such culture system afford a limited growth area, and is not suitable for large scale culture. Microcarrier culture developed in 1967 solved such problem well. Microcarrier means beads with the diameter of $60 \sim 250 \mu \mathrm{m}$ and attachment dependent cells could adhere to its surface, and suspended to grow together with microcarrier in bioreactor. This technology greatly increased surface area of cell growth. Taking microcarrier Cytodex-3 for example, surface area afforded by per $1 \mathrm{~g}$ is $4600 \mathrm{~cm}^{2}$, equal to that of $3 \sim 4$ roller bottles. Moreover, this technology has some dominant advantages: highlight homogenous culture together with the advantages of suspension and attachment, easy to control culture conditions and has a systematic and automatic culture process. In the microcarrier culture of Vero cells with serum free medium, researchers ameliorated attachment effects of cells through the adhesion factor afforded by the culture medium, and on the other hand ameliorated bulk characters of cell adhesion substrates. Earlier microcarrier adopted synthetic polymers such as PVB and PS-TC, and some reports indicated that Vero cells under the condition of serum free were quickly adsorbed and extended. In recent years, many researchers attempted to use natural polymers and their derivatives such as glutin, collagen, fibrin, chitin and etc to prepare microcarrier.

Microcarrier applied extensively is solid ones, including solid sphere and macroporous microcarrier. Solid microcarriers facilitate cells to attach and spread in the bead surface, are conducive to beads to beads inoculation technology in the process of cell infection and amplification imposed by virus, and thus are extensively applied in Vero cell culture, especially for Cytodex series, mainly two types available in the market, namely Cytodex-1and Cytodex-3. Cytodex-1 is used more in the serum free culture of Vero cells because Cytodex-3 is made of collagen which could possibly induce some animal source. When compared to Cytodex 1, Cytodex 3 allowed a 30\%-lower cell concentration and the earlier stationary phase to be obtained, which was probably related to the lower surface area per bead mass of Cytodex 3 microcarriers(de Souza, 2005).

In the condition of low level serum, after Vero cells adsorbed in microcarrier Cytodex-1 for 6 hours, a higher cell density level that was equal to $5 \times 10^{6}$ cells $/ \mathrm{mL}$ was reached with a higher attachment rate of $83 \%$ (Rourou, 2007). Baxter Company has had the case of $6000 \mathrm{~L}$ Vero cell culture to produce influenza virus. Microcarrier technology maintained a high cell population density, but serum free condition facilitates cell apoptosis. Some modifications for Vero cell serum free microcarrier culture includes batch stirring, which facilitate cell attachment(Toriniwa, 2007); Pretreatment with microcarrier, includes changing charge distribution in the surface of microcarrier, which make its surface conducive to cell attachment; Specific trypsin inhibitor was used to terminate protease effects(trypsin could hinder the proliferation of cell in microcarrier) (Genzel, 2006); The addition of shear protectants such as Pluronic F-68 to protein free media is often needed to protect cells against shear forces (could affect downstream process); Silicone pipeline is used in the small bioreactor to eliminate the air bubble when aeration and attenuate cell injury.

\section{(2) Suspension culture}

Due to the high cost of microcarrier, and attempts to continuous high density cell culture, serum free suspension culture of Vero cells has attracted many researchers' attention. The big difference of medium between suspension and attachment culture is Pluronic-F68 or tween-80 with protection effect for fluid mapper shear instead of some attachment factors. As far as suspension is concerned, domestication is crucial, where cells incubated in serum containing medium are transferred into serum free medium. Domestication of serum free medium suspension culture is main optimization 
of serum free medium, which is a project with huge workload. Based on this, changing the growth manner of Vero cells, increasing cell density are conducive to produce vaccine efficiently, extend produce scale and reduce cost. For serum free suspension culture, cells almost need no adaptation period and cell density could reach $0.5-1.0 \times 10^{7}$ cells $/ \mathrm{ml}$ which was far higher than normal attachment culture (5-10 fold). Vero cells in the condition of serum free suspension culture is easy to form all kinds of cell-aggregates of various sizes and adhere to container-wall. Cells in the inner of cell-aggregates were prone to necrosis, and without proper control, culture failure could immediately occur. Marcelo Daelli(Daelli, 2007) was successfully obtained Vero cells by domestication which could single grow in the high density serum free culture not to form mass in USA in 2007. This cell line was preserved by German Collection of Microorganisms and Cell Cultures (DSMZ). The formation of cell-aggregates in suspension culture offers a number of advantages: The cells within the aggregates are at tissue-like densities offering protection against physical stress and possibly helping each other metabolically; The cells are more in vivo-like environment than as monolayers or in single cell suspension cultures; It is easy to change medium with retention because of the fast sedimentation rate of the aggregates. Vero cells were cultured by combing five commercially available base media at different ratios to investigate the effects of serum free medium culture on cell-aggregates. Results showed that cell-aggregates and growth rate by combing different combination of five media varied, and cell density after aggregate could usually reach $10^{6}$ cells $/ \mathrm{ml}$. With the entire serum free medium attempted, a long lag period of between 20 to 30 days was required before the cell concentration reached levels over $10^{6} \mathrm{cells} / \mathrm{ml}$ even though these cells had been adapted to an serum free medium in anchorage-dependent cultures. Serum free suspension culture of Vero cells are not mature and hard to spread. Some researchers inferred that suspension cell could result in block, and protective agents added were mostly abluents which could interfere with downstream process. If cells were not adapted to the serum free medium well, cells grew quite slowly, which could directly ruin the production of virus. Although many disadvantages above, we could speculate that this technology, due to its profound application potential, would be new research direction of large scale culture of Vero cells.

\subsubsection{Culture technology}

Whether attachment or suspension, Vero cell culture could be classified into 3 types as follows:

(1) Batch culture

In batch mode of operation, the medium was not changed during the whole period of culture and virus was added when cell growth attained a certain stage. The entire virus production was harvested when the viral titer was highest. In order to guarantee the growth of virus, Vero cells were usually incubated in serum free medium for several generations to adapt to the medium without serum.

(2) Fed-batch culture

Fed-batch mode of operation was mainly applied for serum free medium suspension culture of Vero cells, as well as microcarrier. $1 / 3$ or $2 / 3$ of the total volume of culture was replaced every day, according to the continuous expenditure and demand of nutrients in Vero cells, which facilitated a high cell density.

(3)Perfusion culture

In perfusion mode of operation, one volume of culture per day was changed by continuous flow medium supplied by a peristaltic pump. This technology could eliminate the toxic metabolites efficiently and promptly, maintain an excellent nutritional status for cells, and thus was desirable for high density culture.

Serum free medium culture of Vero cells are generally classified into the biomass production phase (cell growth phase before virus inoculation) and sometimes also for the virus production phase (period after virus inoculation up to the harvest of virus suspension). According to special requirement of cells and virus during its growth period, different or same substrates or technology were applied. At the first stage, add little serum medium, and eliminate serum containing medium by rinse until cell density attained desired degree. Then add serum free medium for the grown of second stage(Frazzati-Gallina, 2001); Or fed-batch culture was applied at the fist stage, and perfusion culture followed at the second stage, which could attain higher cell density and virus titers compared to the traditional batch culture(Rourou, 2007). This process combination could not only decrease production cost, but also enhance process yield.

\section{Application of serum free medium culture of Vero cells}

Vero cells derived from the African green monkey have been approved for viral vaccine production under specified regulatory guidelines, and are currently used for the production of rabies (Frazzati-Gallina, 2001) and polio vaccines. Moreover, Vero cells have been employed to produce bovine vesicular stomatitis virus, herpes simplex virus, influenza virus, and reovirus (Butler, 2000). The use of these cells also allows large-scale production of vaccines using microcarriers and culture in a bioreactor. For several new eruptible diseases, Vero cells were used as first cell line to produce vaccines. For SARS attracted more attention in past several years, inactivated vaccine have been developed by using Vero cells, and after preclinical investigation, will be undertaken phase 1 clinical trial. As for avian influenza 
H5N1 focused by many researchers, some companies have claimed that H5N1 vaccine derived from Vero cells was of good safety and immunogenicity, and would be undertaken phase 3 clinical trial. Some vaccines were described in detail as follows:

\subsection{Rabies}

The rabies vaccines recommended by WHO include those produced in Vero cells, available since the $80 \mathrm{~s}$ was the most successful. The authors concluded that the results obtained in the present study showed that the new Vero-cell human rabies vaccine, produced by Instituto Butantan, using serum-free medium, was safe and immunogenic. Experimental results showed that dominant commercial serum free medium was properly suitable for cells of virus propagation. Merten have studied that virus was propagated by static culture in spinner flask with serum free medium MDSS2 and attained similar results of cell proliferation with serum containing medium, but higher and earlier the highest virus titers, and 1.5-fold virus production rate. Rabies vaccine cultured in microcarrier Cytodex-3 with serum free medium culture of Vero cells, also attained a higher virus titers. Rabies virus strain production in Vero cells grown on Cytodex 1 in a $2 \mathrm{~L}$ stirred tank bioreactor and in a medium free of components of human or animal origin (VP-SFM) is described. Cell banking procedure in VP-SFM supplemented with an animal components free mixture was reported and cell growth after revitalization was assessed. Vero cells exhibited growth performances (specific growth rate and cell division number) similar to that obtained a higher cell density level maximal virus titer in serum containing medium (Rourou, 2007).

\subsection{Polio vaccines}

There was case that polio vaccines were produced by fixed perfusion culture of Vero cells in a large scale of $1000 \mathrm{~L}$ abroad. Earlier studies indicated that there was no significant difference in static culture between using serum- free or containing medium, and even a higher production rate in serum-free than serum-containing medium. With respect to titers, virus production existed subtle difference using these two mediums.(Cinatl J Jr, 1993), and in general, titers in serum containing medium was little higher. The main difference between two mediums is the rather important difference between virus titers, which is probably due to the different components (age of culture, origin of clones, passage history) of these two mediums which resulted in varied cell physiology and cell metabolism (O.-W. Merten, 1999). This difference could be omitted by subsequent optimization of mediums.

\subsection{Influenza vaccines}

Influenza virus was produce by using chicken embryo for a long time. Since Vero cells were involved in the production of influenza virus, tests have confirmed that almost all virus strains tested in chicken embryos could grow and attain high titers in Vero cells. Although titers were lower than that in chicken embryos, virus yield increased greatly in Vero cells. Several researches found that virus titers in mass production were identical to or higher than in cell monolayer culture, which indicated that the technology of virus production by using Vero cells could be enlarged with no influence on production rate. It is anticipated that scale of producing vaccines in this serum free medium system could attain 1200L. Bacter Company has developed a total virus inactivated influenza vaccine by using Vero cells with serum free medium, which was undertaken phase II/III clinical trial in Europe at present.

\subsection{Enterovirus 71 (EV71) vaccines}

EV71 is a human enterovirus belonging to the Enterovirus genus of the Picornavirus family. At the beginning of 2008, EV71 infection has caused a few outbreaks of hand-foot-and-mouth disease (HFMD) associated with severe neurological diseases in young children in Anhui and Beijing of China. At present, there are no antiviral agents against such virus, and hence developing effective vaccines is the best approach to prevent infection and control the disease. Recent researches have described two VP1 subunit vaccines of EV71, one administered as a DNA vaccine and another as a recombinant protein vaccine, which elicited a neutralization antibody response in both ICR and BALB/c mice. These findings provide direct evidence that VP1 of EV71 contains neutralizing epitopes independent of the other viral capsid proteins, and pave the way for the potential use of VP1 as the backbone antigen for developing subunit vaccines against EV71(Wu CN, 2002). Serum free culture increased cell death rate after infection, reduced the virus specific productivity, but resulted in elicitation of higher neutralizing titers in immunized mice as compared to that parallel obtained in serum-containing cultures. The enhanced immunogenicity of EV71 virions produced in serum-free microcarrier Vero cell culture was in agreement with the result reported for rabies virus vaccine production (Chia-Chyi Liu, 2007). The enhanced immunogenicity of the inactivated virions produced in serum-free cultures is possible that the virions produced in serum-free culturemay give better antigen stability as reported for reovirus.

In recent years, in the area of pharmaceutical engineering vaccines, medImmune Vaccines has engineered a live, attenuated chimeric virus that could prevent infections caused by parainfluenza virus type 3 (PIV3) and respiratory syncytial virus (RSV), causative agents of acute respiratory diseases in infants and young children. Researchers have exerted the development of a serum-free Vero cell culture production platform for this virus vaccine candidate.This improved serum-free process achieved comparable virus titers to the serum-supplemented process, and demonstrated 
consistent results upon scale-up: Vero cultures in roller bottles, spinner flasks and bioreactors reproducibly generated maximum infectious virus titers of $8 \log _{10} \mathrm{TCID}_{50} / \mathrm{ml}$. As it could be speculated, this platform of producing chimeric virus would have great developing potential.

\section{Outlook}

For the issues of cost control and virus yield in large scale culture of cells and vaccines production, we inferred that there were lots of potential to be further developed in serum free medium culture of Vero cells: (1) Considering from Vero cell itself, cell strains of attachment and anti-apoptosis were incubated in the combination of molecular biology and genetic engineering technology, which could greatly ameliorate growth metabolism in serum free condition, and enhance cell resistance ability against harsh environment. For example, genes of adhesion protein, such as vitronectin, introduced into cells, could facilitate cell attachment and extension after the expression of protein; Anti-apoptosis gene Bcl-2, transferred into Vero cells, could effectively inhibit cell apoptosis after expression of protein or high expression of telomerase. (2) Considering from the perspective of mass production, it could facilitate cell growth of high density and hence enhance the production capacity of bioreactors by good combination of serum free medium and bioreactor, and optimization of cell culture processes.

Based on the great application value and prospect of Vero cells, the increment of vaccines requirement in the biological product market, technology of serum free medium culture of Vero cells would be continuously developed.

\section{References}

Andrew, W., \& Udo, R. (1995). Production and properties of a bifunctional fusion protein that mediates attachment of vero cells to cellulosic matrices. Biotechnology and Bioengineering, 47 (2): 147-154.

Butler, M. (2000). Application of a serum-free medium for the growth of VERO cells and the production of reovirus. Biotechnol. Prog, (16): 854-858.

Cinatl, J.J., \& Rabenau, H. (1993). Protein-free culture of Vero cells: a substrate for replication of human pathogenic viruses. Cell Biol Int., 17 (9): 885-895.

Costa, \& Wagner, A. (2007). Immunogenicity and safety of a new Vero cell rabies vaccine produced using serum-free medium. Vaccine, 25: 8140-8145.

Daelli, \& Marcelo (2007). Vero cell line adapted to grow in suspension.

Frazzati, G., \& Neuza M. (2001). Higher production of rabies virus in serum-free medium cell cultures on microcarriers. Journal of Biotechnology, 92: 67 72.

Gao, H.L., \& Wei, C. (2001). Amino Acid Metabolism of Vero Cells in Batch Culture. The Chinese Journal of Process Engineering, 1 (2): 177-180.

Gao, Z.L., \& Zhang, X.P. (2007). Culture of Vero Cells in Serum free and Protein free Medium. Chin J Biologica, 20 (9): 688-690.

Genzel, Y. (2006). Serum-free influenza virus production avoiding washing steps and medium exchange in large-scale microcarrier culture. Vaccine, 24 (2006): 3261-3272.

Inn, H., Yuk, G., \& Lin, B. (2006). A serum-free Vero production platform for a chimeric virus vaccine candidate.Cytotechnology, (51): 183-192.

Kolell, K., \& Padilla, Z. (2005). Virus Production in Vero Cells Using a Serum-Free Medium, JRH. Biosciences.

Litwin, \& Jack (1992). The growth of Vero cells in suspension as cell-aggregates in serum-free media. Cytotechnology, $10: 169-174$.

Liu, C.C., \&Lian, W.C. (2007). High immunogenic enterovirus 71 strain and its production using serum-free microcarrier Vero cell culture. Vaccine, 25 (2007): 19-24.

Merten, O.W., \& Kallel, H. (1999). The new medium MDSS2N, free of any animal protein supports cell growth and production of various viruses. Cytotechnology, 30: 191-201.

Rourou, \& Samia (2007). A microcarrier cell culture process for propagating rabies virus inVero cells grown in a stirred bioreactor under fully animal component free conditions. Vaccine, 25: 3879-3889.

Sebastien, Q., Jacqueline, M., \& Annie, M. (2001). Characterization of Vero cell growth and death in bioreactor with serum-containing and serum-free media. Cytotechnology, 35: 115-125.

Souza, D., \& Marta, C.O. (2005). Influence of Culture Conditions on Vero Cell Propagation on Non-Porous Microcarriers. Brazilian archives of biology and technology.

Toriniwa, \& Hiroko (2007). Japanese encephalitis virus production in Vero cells with serum-free medium using a novel oscillating bioreactor. Biologicals: 1-6. 
Wierzba, A., \& Reichl, U. (1995). Production and properties of a bifunctional fusion protein that mediates attachment of vero cells to cellulosic matrices. Biotechnol Bioeng, Jul 20 (47): 147-154.

Wu, C.N., Lin, Y.C., \& Fann, C. (2002). Protection against lethal enterovirus 71 infection in newborn mice by passive immunization with subunit VP1 vaccines and inactivated virus. Vaccine, 20: 895-904. 